\title{
Phase behaviour of self-assembled monolayers controlled by tuning physisorbed and chemisorbed states: a lattice-model view.
}

\author{
Sara Fortuna \\ MOlecular NAnotechnology for LIfe Science Applications Theory Group, \\ Department of Medical and Biological Sciences, University of Udine, Italy.* \\ David Cheung \\ School of Chemistry, National University of Ireland, \\ Galway, University Road, Galway, Ireland. ${ }^{\dagger}$ \\ Karen Johnston \\ Department of Chemical and Process Engineering, \\ University of Strathclyde, 75 Montrose Street, Glasgow G1 1XJ, U.K. ${ }^{\ddagger}$
}




\begin{abstract}
The self-assembly of molecules on surfaces into $2 \mathrm{D}$ structures is important for the bottom-up fabrication of functional nanomaterials, and the self-assembled structure depends on the interplay between molecule-molecule interactions and molecule-surface interactions. Halogenated benzene derivatives on platinum have been shown to have two distinct adsorption states: a physisorbed state and a chemisorbed state, and the interplay between the two can be expected to have a profound effect on the self-assembly and phase behaviour of these systems. We developed a lattice model that explicitly includes both adsorption states, with representative interactions parameterised using density functional theory calculations. This model was used in Monte Carlo simulations to investigate pattern formation of hexahalogenated benzenes on the platinum surface. Molecules that prefer the physisorbed state were found to self-assemble with ease, depending on the interactions between physisorbed molecules. On the other hand, molecules that preferentially chemisorb, tend to get arrested in disordered phases. However, changing the interactions between chemisorbed and physisorbed molecules affects the phase behaviour. We propose functionalising molecules in order to tune their adsorption states, as an innovative way to control monolayer structure, leading to a promising avenue for directed assembly of novel $2 \mathrm{D}$ structures.
\end{abstract}

PACS numbers:

\footnotetext{
*Electronic address: sara.fortuna@uniud.it; URL: http://www. sarafortuna.eu

$\dagger$ Electronic address: david.cheung@nuigalway.ie

$\ddagger$ Electronic address: karen.johnston@strath.ac.uk
} 


\section{INTRODUCTION}

The self-assembly of functionalised molecules on surfaces is key for the development of novel 2D devices [1] for a variety of applications [2] including graphene and polymer formation [3-5], and semiconductor nanostructures $[6,7]$. Realisation of these applications requires the formation of highly-ordered self-assembled structures on surfaces. The ability of the monolayer to self-assemble and its resulting structure are the result of the delicate interplay between molecule-molecule and molecule-surface interactions. Changing the molecular functionalisation can vary these interactions and, hence, alter the self-assembled structure of the monolayer.

Lattice models are used to provide insight into the interactions that are crucial for controlling the phase behaviour of self-assembled structures. Lattice models have been shown in numerous instances to express a rich phase behaviour, qualitatively reproducing the experimental observations for systems such as dicarboxylic acids [8] or trimesic acid [9, 10] on $\mathrm{Au}(111)$, anthraquinone on $\mathrm{Cu}(111)$ [11], tripod molecules at the liquid/HOPG interface [12] or porphyrin on $\mathrm{Au}(111)$ [13]. Studies of multiple adsorption states include lattice gas models that simulated different adsorption orientations, such as a monolayer of vertically standing diatomic heteronuclear molecules with two distinct adsorption states [14], or homodimers allowed to assume either perpendicular or parallel orientations with respect to the supporting surface [15]. The effect of the surface has been explicitly taken into account in the SANO (Self-Assembly of Nano Objects) model, which relies on precomputed interaction energies based on force fields to predict the self-assembled structures of phthalocyanine derivatives on $\mathrm{Au}(111)$ and $\mathrm{Ag}(111)$ [16].

Self-assembly of halogenated aromatic molecules can be used to form covalently bonded networks, such as brominated tetraphenylporphyrin on $\mathrm{Au}(111)$ [4] and the simpler halogenated benzene on $\mathrm{Cu}(111), \mathrm{Ag}(111)$ or $\mathrm{Au}(111)$ [17]. Halogenation can also influence the self-assembled structures of polyaromatic molecules. For instance, while pentacene forms two coexisting ordered phases on $\mathrm{Ag}(111)$, perfluoropentacene (PFP) forms ordered arrays [18]. Photoemission spectroscopy studies reveal that PFP physisorbs on $\mathrm{Ag}(111)$ [18] but is more weakly physisorbed than pentacene. When these two molecules are deposited on the $\mathrm{Cu}(100)$ surface, the substrate-molecule interaction and molecule orientations are sim-

ilar, however, the pentacene monolayer is disordered whereas the PFP monolayer shows 
long-range crystalline order [19]. Another example is copper-phthalocyanine (CuPc) and perfluorinated $\mathrm{CuPc}$ on $\mathrm{Cu}(100), \mathrm{Cu}(111), \mathrm{Au}(100)$ and $\mathrm{Au}(111)$ surfaces [20]. On $\mathrm{Cu}(100)$ the molecule adsorption and orientation is similar for the two molecules but only the perfluorinated monolayer shows long range order. In contrast, on $\mathrm{Au}(100)$ the two molecules have different orientations on the surface but again only the perfluorinated monolayer shows long range order, although the patterns are different to those on copper. This different behaviour was attributed to relative differences in molecule-surface or molecule-molecules interactions, where the molecule-surface chemical interaction is stronger on copper than on gold, and hence on gold van der Waals molecule-molecule interactions are expected to dominate [20]. Other studies of halogenated polyaromatic molecules on $\mathrm{Si}(111)$ showed that the subtle balance between molecule-molecule and molecule-surface interactions led to the formation of open or compact networks depending on the end groups [21-23].

Although self-assembly of aromatic molecules has been demonstrated numerous times on surfaces including gold, silver and copper, ordered aromatic monolayers have not been observed on platinum. For example, benzene, naphthalene and naphthoquinones form ordered monolayers on $\operatorname{Rh}(111)$ but not on $\operatorname{Pt}(111)$ [24-26]. In the case of platinum, density functional theory (DFT) calculations have shown that halogenated benzene molecules have two well-defined adsorption states: a physisorbed state and a chemisorbed state [27, 28]. The relative stability of these states can be tuned by halogenation and this raises the question whether halogenation can be used to induce self-assembled structures on platinum; however, this effect has not yet been explored.

In this work, we study the effect of the interplay between chemisorbed and physisorbed states on the self-assembly of halogenated benzene molecules on platinum by using a $2 \mathrm{D}$ hexagonal lattice model we have recently developed [8]. Our model has been extended to include chemisorbed and physisorbed states, with representative interactions parameterised by DFT calculations. The inclusion of both adsorption states, which has not been accounted for in previous lattice models, is necessary to capture the essential phenomenon for the prediction of ordering and phase transitions of aromatic molecules on platinum. The model we propose assumes that molecules exist in one of two discrete orientations with respect to the lattice and one of two allowed adsorption states, namely chemisorbed or physisorbed. While this discretization of the configurational space characterizes this as a discrete lattice model, the inclusion of different adsorption states is an additional level of complexity with 
respect to traditional lattice models. Monte Carlo (MC) simulations allow the time and length scales necessary for observing the self-organisation process to be reached. The novelty of the approach is in studying the collective behaviour and phase transitions of monolayers at surfaces with both chemisorbed and physisorbed adsorption states.

This study is the first application of a lattice model to the self-assembly of halogenated aromatic molecules on the platinum surface. In the following we first describe the lattice model (Sec. IIA) and its interactions that involve parameterisation from DFT calculations (Sec. IIB), then we will describe the MC simulations (Sec. IIC) and discuss the emerging self-assembled patterns (Sec. IIIB). We found that using halogenation to change the relative stability of the chemisorption/physisorption state strongly affects the ordering of the monolayer and their phase transition behaviour. This provides a new route to controlling monolayer stability on platinum, and our model can guide development and formation of novel graphene structures.

\section{METHOD}

\section{A. Hexagonal Model}

We simulate the self-assembly of $N$ non-overlapping hexagonal molecules on an hexagonal lattice in the NVT ensemble. The system is discretized so that each lattice site can be occupied by only one molecule, as shown in Fig. 1. Lattice sites therefore do not represent a particular group of atoms on the supporting surface, but they rather indicate possible discrete molecular positions on the surface. The lattice is hexagonal to take into account both the molecular symmetry and their possible hexagonal molecular packing, as formerly done with other aromatic molecules [8].

On the lattice, each molecule can switch between two states: a chemisorbed state and

a physisorbed state. Molecules interact with the surface with $E_{\text {ads }}^{\text {phys }}$ when physisorbed and $E_{\text {ads }}^{\text {chem }}$ when chemisorbed. Our model does not take into account multiple adsorption configurations for the physisorbed and chemisorbed states and in this study we represent the surface-molecule interaction for each state by their most energetically favorable configuration, and take these values from a former DFT study [28]. Molecules in both adsorption states can be oriented with their $\mathrm{C}-\mathrm{C}$ axes either aligned with the horizontal axes and 


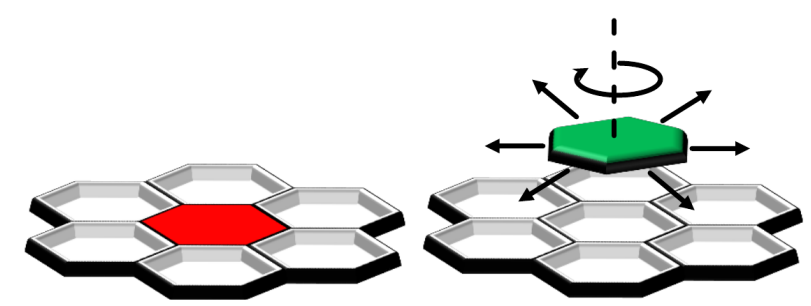

(a) chemisorbed

(b) physisorbed

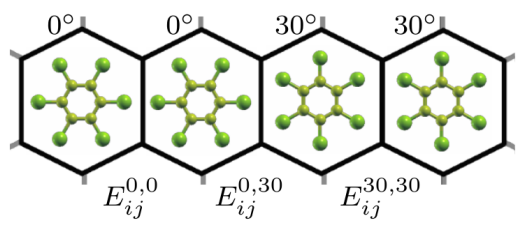

(c) molecule-molecule interactions

FIG. 1: Possible states on the hexagonal lattice. The molecule can be either (a) chemisorbed or (b) physisorbed. The molecule can freely move on the surface only when physisorbed. For each adsorption state molecules can have two possible orientations (either $0^{\circ}$ or $30^{\circ}$ ) and, therefore, three possible molecule-molecule interactions (c).

pointing towards a hexagonal lattice edge $\left(0^{\circ}\right)$ or pointing towards a hexagonal lattice node $\left(30^{\circ}\right)$, as shown in Fig. 1(c). Adjacent molecules interact with each other via van der Waals interactions, which depend on the relative orientations of the adjacent molecules. In the lattice model the corresponding molecule-molecule interaction parameters, as indicated in Fig. 1(c), are:

- $E_{i j}^{0,0}$ when their $\mathrm{C}-\mathrm{C}$ axes are aligned

- $E_{i j}^{0,30}$ when one molecule is tilted in the $x y$-plane with respect to each other

- $E_{i j}^{30,30}$ when the molecules have parallel C-C axis and two carbon atoms point towards each other

The number of molecules is fixed on the lattice. For the chemisorption state the energies vary significantly on different surface sites, hindering surface diffusion, whereas for the physisorbed states the molecules are relatively insensitive to the surface site [28, 29]. Therefore, in our model, molecules can translate and rotate freely only when physisorbed, whereas, when chemisorbed they are anchored to their position and orientation and can only change their adsorption state. Every simulation step corresponds to a Monte Carlo step in which a tile is selected at random and either a change in adsorption state, a translation to an adjacent lattice site, or a rotation is attempted. Translations and rotations are always 
rejected if the tile is chemisorbed, and attempted translations to already occupied sites are also rejected. The other possible moves are accepted or rejected following the Metropolis acceptance probability [30].

The overall Hamiltonian of the system is:

$$
H=\sum_{<i>} E_{\mathrm{ads}}^{\mathrm{s}_{i}}+\frac{1}{2} \sum_{<i j>} k_{\mathrm{s}_{i}, \mathrm{~s}_{j}} E_{i j}^{l_{i}, l_{j}}
$$

where $\mathrm{s}_{i}$ is the adsorption state of each molecule $i, l_{i}$ and $l_{j}$ are the orientation of the molecules $\mathrm{i}$ and $\mathrm{j}$, respectively, and the summation $\langle i\rangle$ is taken over all the tiles and the summation $\langle i j\rangle$ is over adjacent tiles. $k_{\mathrm{s}_{i}, \mathrm{~s}_{j}}$ is a parameter that adjusts the side-side interactions between adjacent molecules, which is introduced to study the possible effects of different adsorption states on the molecule-molecule interaction.

\section{B. System Parameterisation}

The lattice model was applied to $\mathrm{C}_{6} \mathrm{H}_{6}, \mathrm{C}_{6} \mathrm{~F}_{6}, \mathrm{C}_{6} \mathrm{Cl}_{6}$, and $\mathrm{C}_{6} \mathrm{Br}_{6}$ monolayers on the $\operatorname{Pt}(111)$ surface and the interactions were parameterised using the results from density functional calculations [28]. The model requires the interactions between the molecule and the surface and between neighbouring molecules. Although hexaiodobenzene also exhibits bistable adsorption states on platinum, it is likely that it would dissociate before reaching the chemisorbed state [31] and hence it has not been considered in this study.

\section{Molecule-Surface Interactions}

The interaction between halogenated benzene molecules and the Pt(111) surface has been previously investigated in the literature [28], and we use these adsorption energies in our model. These results were calculated using the vdW-DF functional with PBE exchange, which was found to give improved results for molecule-surface interactions. The adsorption energies for both long-range (physisorbed) and short-range (chemisorbed) structures were reported and a crossover in stability as the number of halogen atoms is increased was observed. The reported adsorption energies for the hexalogenated benzene derivatives here investigated are given in Tab. I. 
We stress here that there is no significant change in the structure of the molecule between the physisorbed and chemisorbed states. For chemisorption on silicon, it is known that benzene rings can form tight-bridge or butterfly structures $[32,33]$, where the ring undergoes significant distortion from sp2 to $s p 3$ bonding. However, on platinum the ring does not distort and remains sp2-bonded. Instead, the structures differ in the relative heights of the halogen atoms to the ring [28]. For the physisorbed structure the larger halogen atoms bend down toward the surface and the ring remains at a height of 3.1-3.4 $\AA$ from the surface. For the chemisorbed structure the ring moves closer to the surface at a height of $2.1 \AA$ and the halogen atoms bend away from the surface. The size of the molecule in the two states is relatively unchanged and therefore the lattice model can treat the two states with the same tile size and shape.

It is known that halogenated benzene molecules on platinum have several possible adsorption sites, orientations and energies [28]. In this study we assume that for both adsorption states the lowest energy adsorption configuration is dominant. Hence, we represent the surface-molecule interactions for the physisorbed and chemisorbed states by their most energetically favorable configuration and take the interaction energies from an earlier DFT study [28]. For difluorobenzene and hexafluorobenzene the stable configuration for the chemisorbed site was adsorption on the bridge site with a $30^{\circ}$ angle with the crystal axis. For all molecules we have taken the bridge $30^{\circ}$ adsorption energies to be the parameters for the molecule-surface interaction.

\section{Molecule-Molecule Interactions}

The molecule-molecule interactions are not given in the literature and, therefore, they were here calculated using density functional calculations. We note that the moleculemolecule interaction will be different between molecules in different adsorption states, but for the physisorbed state the molecular structures are very similar to their gas structures and we can obtain reasonably good estimate for the interaction between two physisorbed molecules by calculating the interaction between two gas state molecules, described below.

Density functional theory calculations were performed using the QUANTUM ESPRESSO code [34]. The vdW-DF exchange and correlation functional [35-37] was used, which accounts for van der Waals interactions and was found to be optimal for interactions between 


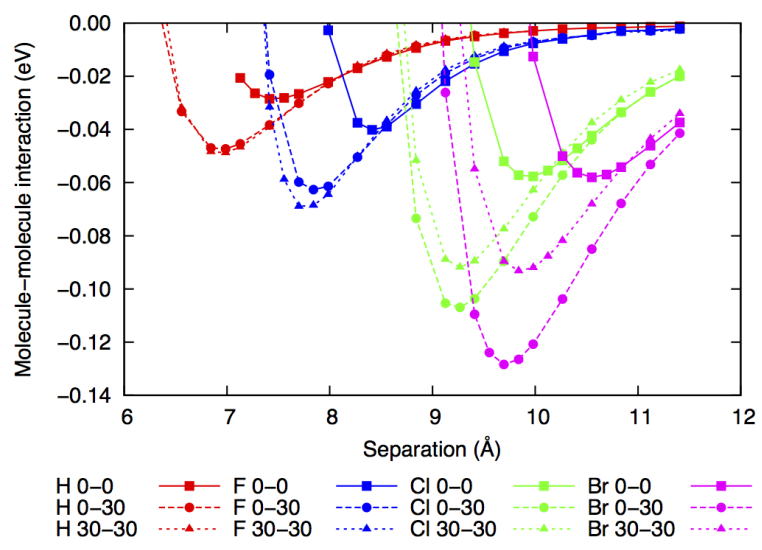

FIG. 2: Interaction energy between two gas molecules from DFT calculations.

benzene dimers [38]. The molecule-surface interaction energies taken from literature [28] were also calculated using the vdW-DF functional but they used PBE for the exchange functional, which was found to give better results for molecule-surface interactions. Valence electrons are represented using a plane wave basis and core electrons are represented with projector augmented waves. The wavefunction and charge density cutoff energies were 70 Ry and 280 Ry, respectively, for $\mathrm{C}_{6} \mathrm{H}_{6}, \mathrm{C}_{6} \mathrm{Cl}_{6}$ and $\mathrm{C}_{6} \mathrm{Br}_{6}$ and $80 \mathrm{Ry}$ and $320 \mathrm{Ry}$, respectively, for $\mathrm{C}_{6} \mathrm{~F}_{6}$. These cutoffs were chosen so that the total energy per atom was converged to approximately $1 \mathrm{mRy}$. The calculations were performed with a single $k$-point.

Two molecules were placed in a tetragonal box, with periodic boundary conditions in $x, y$ and $z$. One molecule was centred on $x=0$ and the other at $x=0.5$ in units of the box length. The box length in the $y$ and $z$-directions were fixed at $26.9 \AA$ and $21.6 \AA$, respectively, so that molecule-molecule interactions in the $y$ and $z$ directions were negligible. The length of the box along the $x$-direction was varied. The calculated intermolecular energy was then divided by two to obtain the interaction energy. This was repeated for molecules with a $0^{\circ}$ and a $30^{\circ}$ angles.

The physisorbed-physisorbed (or gas-gas) interaction energies vs the distance between the centres of geometry of the molecules are shown in Fig. 2. The location of the interaction minima depends on the molecular size, which increases from $\mathrm{C}_{6} \mathrm{H}_{6}$ to $\mathrm{C}_{6} \mathrm{Br}_{6}$. In addition the position of the minimum also depends on the orientation of the molecules. For all molecules, the $0-0$ interaction minimum is located at a larger intermolecular distance than the 0-30 and 30-30 interaction minima, whose minimum distances almost overlap. However, the difference 


\begin{tabular}{|l|cc|ccc|}
\hline Molecule & $E_{\text {ads }}^{\text {chem }}$ & $E_{\text {ads }}^{\text {phys }}$ & $E_{i j}^{0,0}$ & $E_{i j}^{0,30}$ & $E_{i j}^{30,30}$ \\
\hline $\mathrm{C}_{6} \mathrm{H}_{6}$ & -1.61 & -1.03 & -0.03 & -0.05 & -0.05 \\
$\mathrm{C}_{6} \mathrm{~F}_{6}$ & -1.37 & -1.02 & -0.04 & -0.06 & -0.07 \\
$\mathrm{C}_{6} \mathrm{Cl}_{6}$ & -0.85 & -1.56 & -0.06 & -0.11 & -0.09 \\
$\mathrm{C}_{6} \mathrm{Br}_{6}$ & -1.67 & -1.89 & -0.06 & -0.13 & -0.09 \\
\hline
\end{tabular}

TABLE I: Simulation parameters for the molecule-surface interactions are from DFT calculations in Ref. [28]. The physisorbed-physisorbed intermolecular interactions correspond to the minima in Fig. 2. All values are in $\mathrm{eV}$.

in the positions of the minima due to rotations is small compared to the size of the molecule so these size differences have been neglected in the lattice model.

The physisorbed-physisorbed interactions become stronger from $\mathrm{C}_{6} \mathrm{H}_{6}$ to $\mathrm{C}_{6} \mathrm{Br}_{6}$. For all molecules, the 0-0 interaction is the least favourable. Benzene does not have a strong interaction between molecules and the interaction between $0-30$ and 30-30 orientations is almost identical. $\mathrm{C}_{6} \mathrm{~F}_{6}$ has a slightly deeper minimum for the 30-30 interaction, than for the 0-30 interaction. The two larger molecules, namely $\mathrm{C}_{6} \mathrm{Cl}_{6}$ and $\mathrm{C}_{6} \mathrm{Br}_{6}$, prefer the 0-30 orientation. However, the energetic differences are small and comparable to $k_{\mathrm{B}} T\left(k_{\mathrm{B}} T \sim\right.$ $0.025 \mathrm{eV}$ at temperature $T=298 \mathrm{~K})$.

Obtaining the interaction between two chemisorbed molecules or between a chemisorbed and a physisorbed molecule is computationally demanding. Rather than calculating these directly, we instead treat the interactions as being proportional to the physisorbedphysisorbed interaction energy but scaled by an adjustable parameter, $k_{\text {chem,chem }}$ between two chemisorbed molecules or $k_{\text {phys,chem }}$ between a physisorbed and a chemisorbed molecule.

The full set of parameters for the lattice model is shown in Tab. I. The molecule-surface interactions were taken from the literature [28], as described in the previous subsection, and the minima of the intermolecular interactions, in Fig. 2 are taken as the physisorbedphysisorbed intermolecular interactions.

\section{Monte Carlo Simulations}

We set up a system composed of $N=1025$ molecules interacting via the Hamiltonian of Eq. 1 with the parameters of Tab. I on a $50 \times 50$ hexagonal lattice with periodic boundary conditions. We build a chain of 200 simulations, each simulation consisting of $5000 \mathrm{~N}$ equili- 


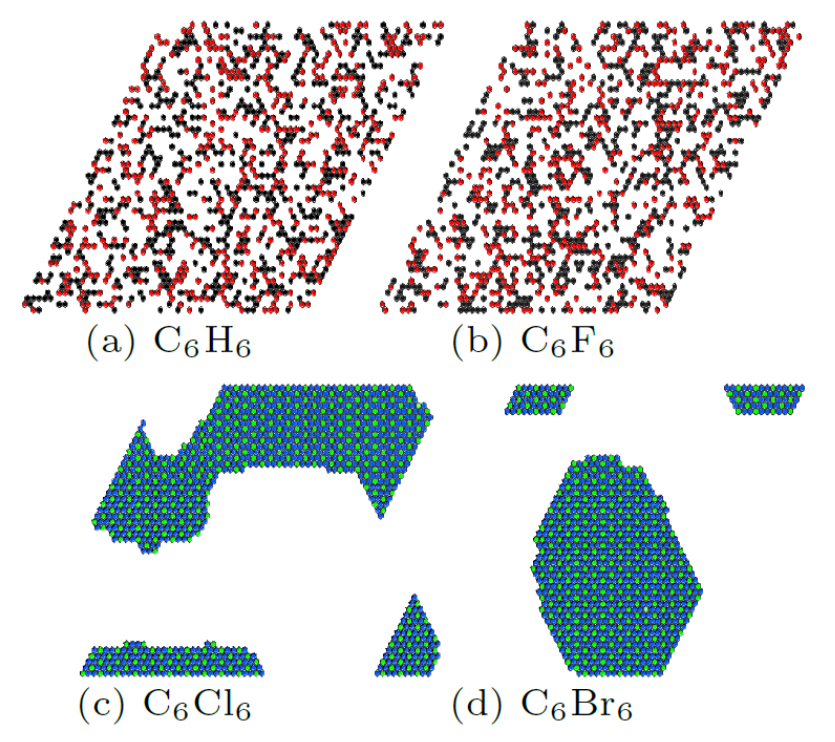

FIG. 3: Low temperature configurations with $k_{\text {phys,phys }}=k_{\text {phys,chem }}=k_{\text {chem,chem }}=1.0$, where red/black molecules are chemisorbed, blue/green are physisorbed, and black/blue molecules are rotated by $30^{\circ}$ with respect to the red/green ones.

bration steps followed by $5000 \mathrm{~N}$ production steps, sufficient to guarantee both the equilibration of the system and an adequate sampling of the configurational space. Every simulation chain starts at $k_{\mathrm{B}} T=0.2 \mathrm{eV}$ (as the Boltzmann constant $k_{\mathrm{B}}=8.6173324 \times 10^{-5} \mathrm{eV} / \mathrm{K}$ this corresponds to a temperature of $\sim 2320 \mathrm{~K}$ ) and every successive simulation is run by decreasing the temperature by $k_{\mathrm{B}} \Delta T=0.001 \mathrm{eV}$ and by using the last configuration of the former simulation as the starting configuration. Configurations are sampled every $N$ production steps and the simulations are run in 10 replicas. All order parameter averages $\langle\ldots\rangle$ are ensemble averages further averaged over the 10 replicas.

\section{RESULTS AND DISCUSSION}

\section{A. Molecular Organisation}

On the lattice, by annealing the system using the parameters of Tab. I and assuming $k_{\text {phys,chem }}=k_{\text {chem,chem }}=k_{\text {phys,phys }}=1.0$, we obtain the low energy configurations of Fig. $3 . \mathrm{C}_{6} \mathrm{H}_{6}$ and $\mathrm{C}_{6} \mathrm{~F}_{6}$ form chemisorbed disordered arrested phases (Fig. 3a,b), while $\mathrm{C}_{6} \mathrm{Cl}_{6}$ and $\mathrm{C}_{6} \mathrm{Br}_{6}$ form an ordered physisorbed packed pattern (Fig. 3c,d). The origin of this different phase behaviour can be understood by looking at the process leading to their formation. 

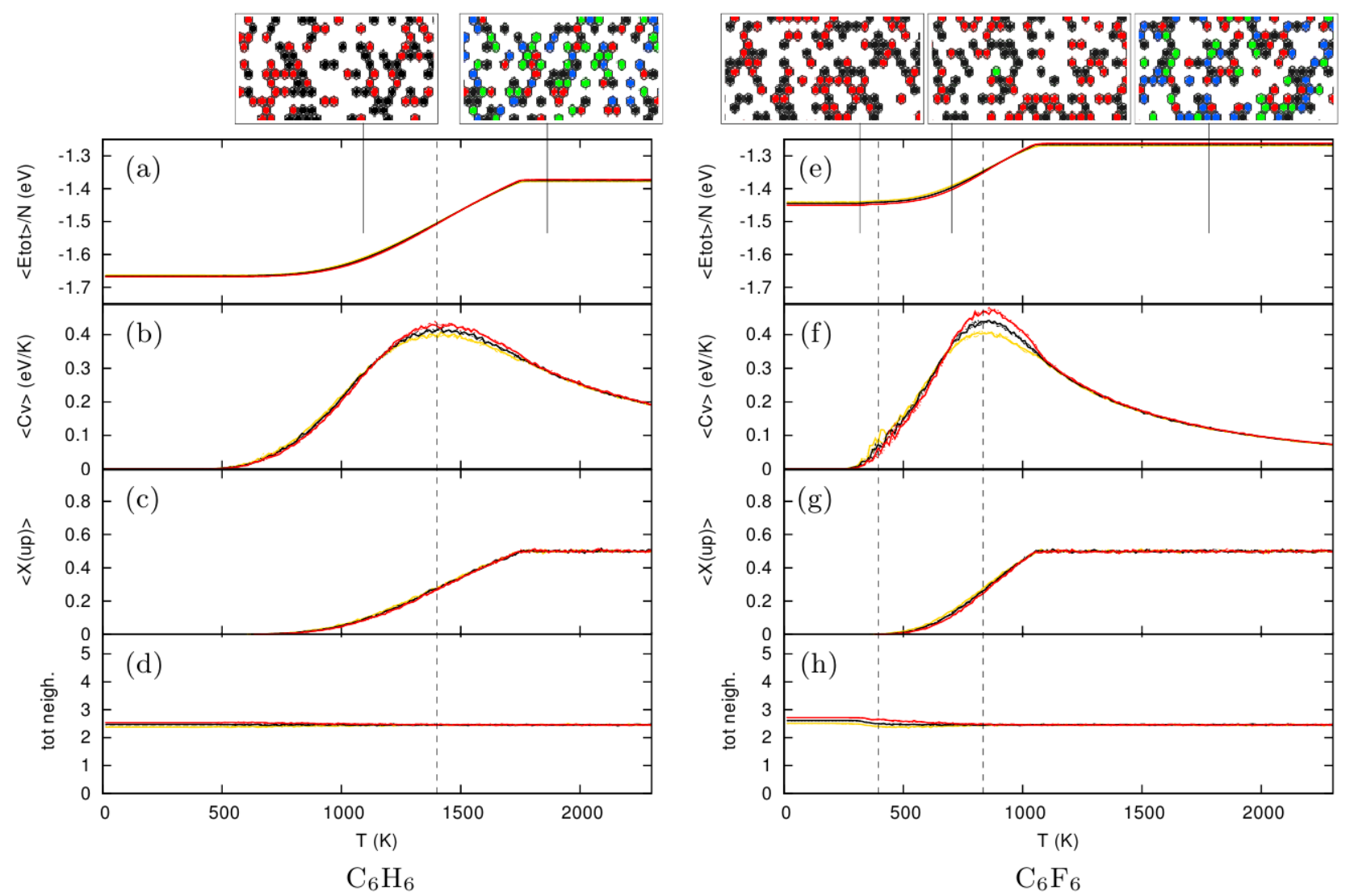

FIG. 4: Comparisons with all parameters as in Tab. I for $k_{\text {phys,chem }}=0.9$ (red), 1.0 (black), 1.1 (yellow). Only the results with $k_{\text {chem,chem }}=1.0$ (solid lines) are visible since they overlap with the $k_{\text {chem,chem }}=0.9$ (dashed), 1.1 (dotted) curves. In all the cases $k_{\text {phys,phys }}=1.0$. Vertical lines are associated with structural transitions. The insets show the patterns observed at the indicated temperatures, red/black molecules are chemisorbed, blue/green are physisorbed, and black/blue molecules are rotated by $30^{\circ}$ with respect to the red/green ones.

To characterise the phases encountered and identify the transitions between them, we look at a number of order parameters: total system energy $E_{\text {tot }}$ given by the hamiltonian of Eq. 1, the heat capacity at constant volume $C_{V}$ calculated with the fluctuation formula $k_{\mathrm{B}} T^{2} C_{V}=\left\langle\left(E_{\mathrm{tot}}-\left\langle E_{\mathrm{tot}}\right\rangle\right)^{2}\right\rangle \equiv\left(\Delta E_{\mathrm{tot}}\right)^{2}$, the fraction of physisorbed molecules $\chi_{\mathrm{up}}$, and the number of neighbours shown as a function of temperature, all indicated by black solid lines in Fig. 4, Fig. 5, and Fig. 6. At high temperature all systems are disordered, with an equal amount of physisorbed and chemisorbed molecules and an average number of neighbours $6 \times 1025 / 2500$ (maximum number of neighbours times the number density). As can be seen the different systems can be divided into two groups, depending on whether $E_{\text {ads }}^{\text {chem }}<E_{\text {ads }}^{\text {phys }}$ $\left(\mathrm{C}_{6} \mathrm{H}_{6}\right.$ and $\mathrm{C}_{6} \mathrm{~F}_{6}$, Fig. 4) or vice-versa $\left(\mathrm{C}_{6} \mathrm{Cl}_{6}\right.$ and $\mathrm{C}_{6} \mathrm{Br}_{6}$, Fig. 5). 

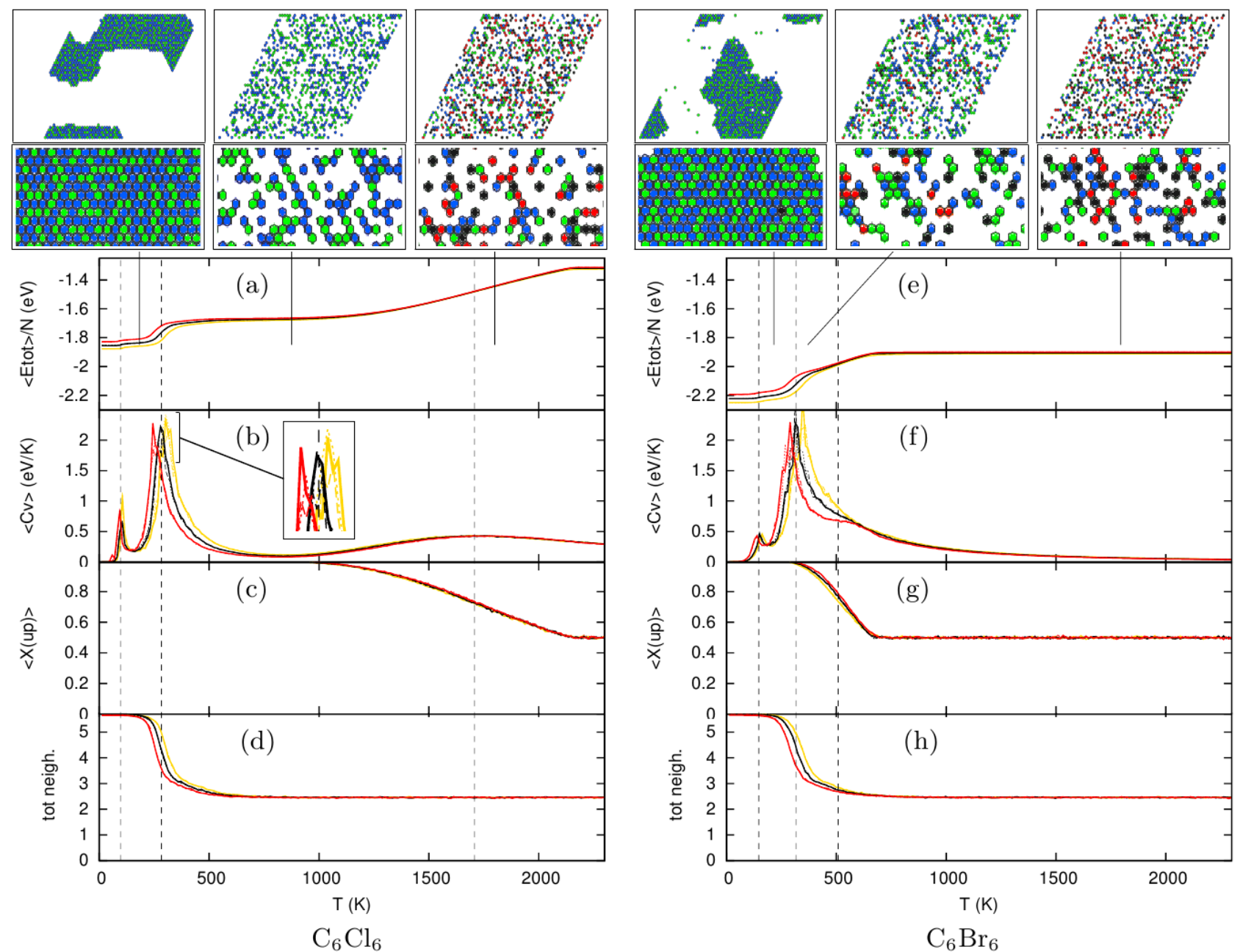

FIG. 5: Comparisons with all parameters as in Tab. I for $k_{\text {phys,chem }}=0.9$ (red), 1.0 (black), 1.1 (yellow). The inset in panel (b) shows a $\times 2$ magnification of the highlighted area of the plot. Only the results with $k_{\text {chem,chem }}=1.0$ (solid lines) are visible since they overlap with the $k_{\text {chem,chem }}=0.9$ (dashed), 1.1 (dotted) curves. In all the cases $k_{\text {phys,phys }}=1.0$. Vertical lines are associated with structural transitions. The insets show the patterns observed at

the indicated temperatures, red/black molecules are chemisorbed, blue/green are physisorbed, and black/blue molecules are rotated by $30^{\circ}$ with respect to the red/green ones.

For $\mathrm{C}_{6} \mathrm{H}_{6}$ and $\mathrm{C}_{6} \mathrm{~F}_{6}$ there is only one well-defined transition corresponding to a change from a disordered mixed phase to a disordered chemisorbed phase (denoted by the peak in the heat capacity, Fig. 4a,e). The molecules have a very close $E_{\text {ads }}^{\text {phys }}(-1.03 \mathrm{eV}$ and $-1.02 \mathrm{eV})$, but due to the much larger $E_{\text {ads }}^{\text {chem }}$ for $\mathrm{C}_{6} \mathrm{H}_{6}(-1.61 \mathrm{eV})$ with respect to that of $\mathrm{C}_{6} \mathrm{~F}_{6}(-1.37 \mathrm{eV})$, the transition occurs at higher temperature $(1400 \mathrm{~K}$ vs $800 \mathrm{~K})$ for $\mathrm{C}_{6} \mathrm{H}_{6}$ than for $\mathrm{C}_{6} \mathrm{~F}_{6}$. The low temperature phases remain disordered since the chemisorbed molecules cannot move on 

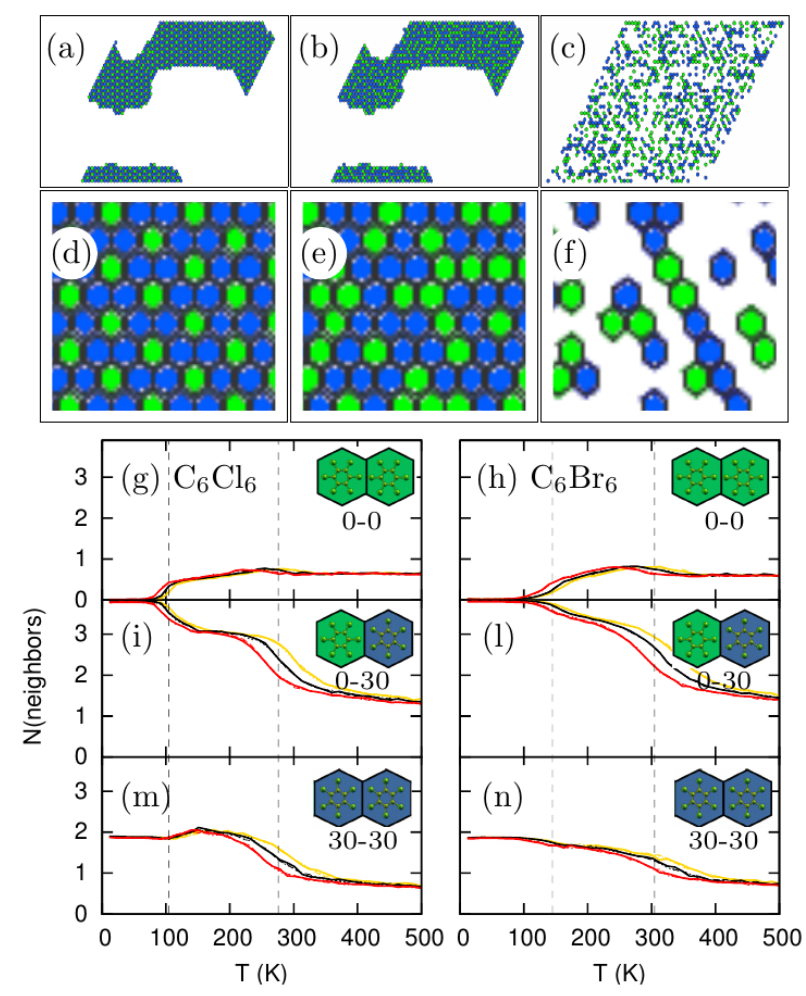

FIG. 6: Simulation snapshots at (a) 0K, (b) 200K, (c) $500 \mathrm{~K}$ for $\mathrm{C}_{6} \mathrm{Cl}_{6}$ with $k_{\text {phys,chem }}=k_{\text {chem,chem }}=1.0$, where blue molecules are rotated by $30^{\circ}$ with respect to the green ones. (d-f) magnification of the above snapshots. (g-n) Orientational order for $\mathrm{C}_{6} \mathrm{Cl}_{6}$ and $\mathrm{C}_{6} \mathrm{Br}_{6}$, comparisons with all parameters as in Tab. I for $k_{\text {phys,chem }}=0.9$ (red), 1.0 (black), 1.1 (yellow). Only the results with $k_{\text {chem,chem }}=1.0$ (solid lines) are visible since they overlap with $k_{\text {chem,chem }}=0.9$ (dashed), 1.1 (dotted) curves. In all the cases $k_{\text {phys,phys }}=1.0$. Vertical lines are associated with structural ransitions.

the surface and do not have the energy to escape back into the mobile physisorption state. The behaviour of both $\mathrm{C}_{6} \mathrm{H}_{6}$ and $\mathrm{C}_{6} \mathrm{~F}_{6}$ are very similar, with $\mathrm{C}_{6} \mathrm{~F}_{6}$ showing slightly more order, evidenced by the slightly higher number of neighbours (Fig. 4d,h) and the appearance of small features in the $C_{V}$ at about $400 \mathrm{~K}$ (Fig. 4f) .

For comparison, experimental results on $\operatorname{Pt}(111)$ are available only for benzene, which has been shown to form disordered patterns [24, 25]. However, the qualitative behaviour of the explored systems is similar to perfluorinated pentacene on $\mathrm{Cu}(100)[19,39]$, and copper phthalocyanine on $\mathrm{Cu}(100), \mathrm{Au}(100)$ and $\mathrm{Au}(111)$ [20], in that fluorination resulted in a more ordered monolayer.

$\mathrm{C}_{6} \mathrm{Cl}_{6}$ and $\mathrm{C}_{6} \mathrm{Br}_{6}$ show a richer phase behaviour. The high temperature phase transition at $1700 \mathrm{~K}$ for $\mathrm{C}_{6} \mathrm{Cl}_{6}$ is from a disordered mixed phase to a disordered physisorbed phase and is shown in Fig. 5a. At much lower temperatures two distinct events occur (Fig. 6a): first, 


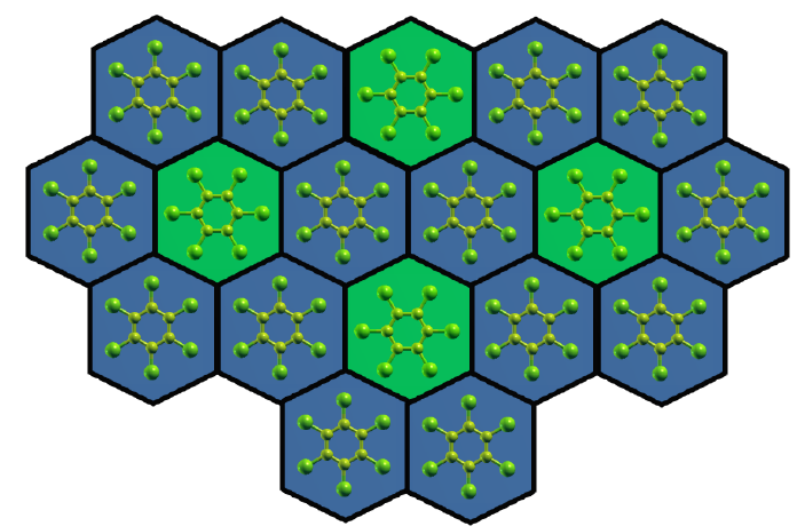

FIG. 7: Ordered pattern observed for $\mathrm{C}_{6} \mathrm{Cl}_{6}$ and $\mathrm{C}_{6} \mathrm{Br}_{6}$.

at $270 \mathrm{~K}$, the system packs by increasing the number of favourable neighbours (0-30 and 30-30), then, at $100 \mathrm{~K}$, it forms an ordered pattern by maximising the most favourable 0-30 interactions, quenching the 0-0 interactions and reducing the number of 30-30 interactions keeping only those necessary to maximise the 0-30 ordering. The low temperature pattern is shown in Fig. 7, where most of the molecules are oriented at $30^{\circ}$ with the lattice forming a honeycomb pattern kept together by 30-30 interactions with a central $0^{\circ}$-oriented molecule forming 0-30 interactions with the surrounding molecules

The energy gap between physisorbed and chemisorbed states is smaller for $\mathrm{C}_{6} \mathrm{Br}_{6}$ than for $\mathrm{C}_{6} \mathrm{Cl}_{6}$ moving the transition between the disordered mixed phase and the disordered chemisorbed phase towards a much lower temperature than that for $\mathrm{C}_{6} \mathrm{Cl}_{6}$ (Fig. 5g). This causes the high temperature transition to overlap with the packing transition (Fig. 5h), forming a shoulder at $500 \mathrm{~K}$ in the $C_{V}$ (Fig. 5f). At $300 \mathrm{~K}$, similar to that observed for the former system, molecules pack once again by first increasing the number of 0-30 and 30-30 neighbours (Fig. 6b), then organising into the same honeycomb ordered pattern expressed by $\mathrm{C}_{6} \mathrm{Cl}_{6}$ and shown in Fig. 7 following a similar mechanism.

Orientational order is reached for $\mathrm{C}_{6} \mathrm{Br}_{6}$ and $\mathrm{C}_{6} \mathrm{Cl}_{6}$ via two different mechanisms (Fig. 6). In $\mathrm{C}_{6} \mathrm{Cl}_{6}$ the 0-30 interaction $(-0.11 \mathrm{eV})$ and the $30-30$ interactions $(-0.09 \mathrm{eV})$ are quite comparable: the system packs by increasing the number of favourable neighbours (0-30 and 30-30), then it crystallises by maximising the 0-30 interactions and reducing the number of 0-30 interactions keeping only those necessary to maximise the 0 -30 ordering. For the $\mathrm{C}_{6} \mathrm{Br}_{6}$, the 30-30 interaction has the same magnitude as that of $\mathrm{C}_{6} \mathrm{Cl}_{6}$ while the $0-30$ is stronger $(-0.13 \mathrm{eV})$. In that case both the 0-30 and 30-30 neighbours simply increase monotonically 
as the temperature decreases.

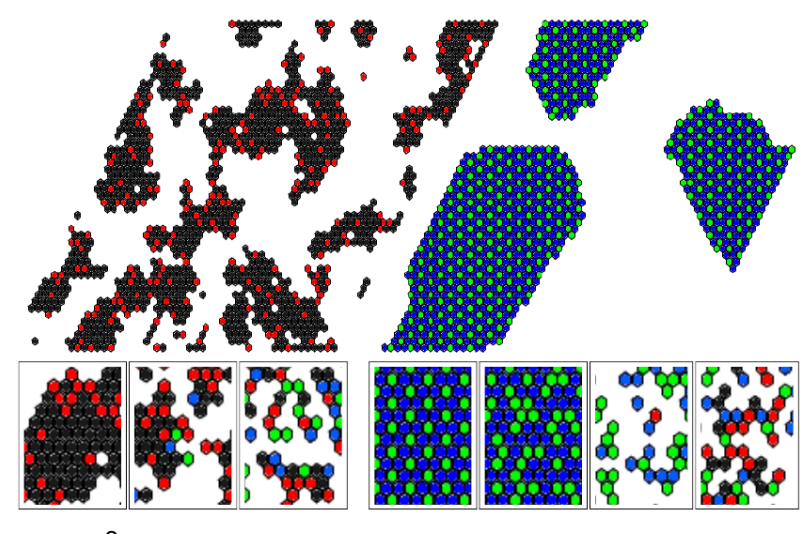

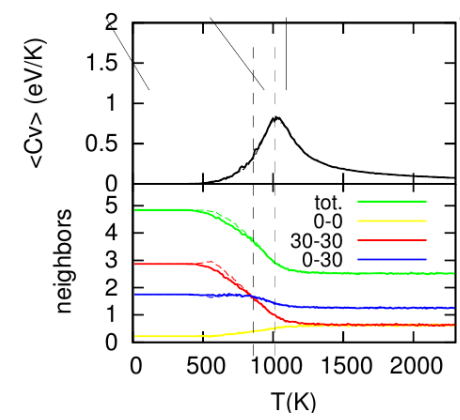

(a) $\mathrm{C}_{6} \mathrm{~F}_{6}$

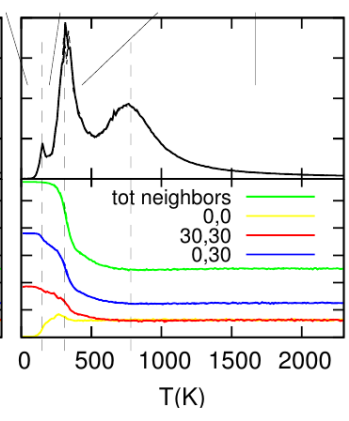

(b) $\mathrm{C}_{6} \mathrm{Br}_{6}$

FIG. 8: Low energy configurations and patterns formed when $k_{\text {phys,phys }}=k_{\text {chem,chem }}=1.0$, $k_{\text {phys,chem }}=0.0$, and the corresponding order parameters obtained by decreasing (solid) and increasing (dashed) temperature.

\section{B. Molecule-molecule interactions}

Up to now we have assumed all the side-side interactions to be unaffected by their adsorption state. The side-side interactions have been calculated in vacuum and we have assumed that these represent well the interaction between two physisorbed molecules whose electronic structure is only slightly perturbed by the presence of the surface. We now investigate the possible effect of the chemisorption on the side-side interactions by setting $k_{s i, s j}$ to $0.9,1.0$ and 1.1 when one molecule is chemisorbed. This is done for both $k_{\text {phys,chem }}$ and $k_{\text {chem,chem }}$, while $k_{\text {phys,phys }}$ is fixed at 1.0. It emerges that the onset of the observed structural transitions is ruled by $k_{\text {phys,chem }}$ as three distinct line bundles (red, black, yellow) are observed in all the plots of Fig. 4, Fig. 5, and Fig. 6, while lines at different $k_{\text {chem,chem }}$

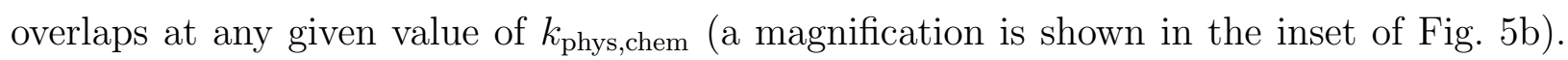


Tuning the interactions between two chemisorbed molecules has a negligible effect and the associated curves simply overlap forming a single bundle when $k_{\text {chem,chem }}$ is tuned at constant $k_{\text {phys,chem }}$.

Tuning instead $k_{\text {phys,chem }}$ affects the $C_{V}$ curve with the most visible effect being that of favoring the packing of $\mathrm{C}_{6} \mathrm{H}_{6}$ and $\mathrm{C}_{6} \mathrm{~F}_{6}$ low temperature structures when the interaction strengths decreases as is seen for $k_{\text {phys, chem }}=0.9$ compared to 1.1 (compare red with yellow

lines in Fig. 4). Another effect of increasing $k_{\text {phys, chem }}$ is that of moving the packing and the ordering transitions for $\mathrm{C}_{6} \mathrm{Cl}_{6}$ and $\mathrm{C}_{6} \mathrm{Br}_{6}$ towards higher temperatures and the physisorption/chemisorption transition towards lower temperatures (yellow lines in Fig. 5). In the extreme case in which $k_{\text {phys, chem }}$ is completely switched off (Fig. 8), $\mathrm{C}_{6} \mathrm{H}_{6}$ and $\mathrm{C}_{6} \mathrm{~F}_{6}$ pack by forming connected islands, which are larger for $\mathrm{C}_{6} \mathrm{~F}_{6}$ and dominated by 30-30 interactions (Fig. 8a), whereas for $\mathrm{C}_{6} \mathrm{Br}_{6}$ the packing interactions appear as a distinct peak at $750 \mathrm{~K}$ (Fig. 8b).

We note again that the molecule-molecule interactions are calculated using a different vdW functional than the molecule-surface interactions. vdW functionals are known to give slightly different energies, however, even if the vdW-DF does not describe the moleculemolecule interactions perfectly, this result has shown that small differences in interaction energies between the molecules do not significantly affect the packing or phase behaviour.

\section{CONCLUSIONS}

The phase behaviour and self-assembled patterns formed by hexahalogenated molecules on a $\mathrm{Pt}(111)$ surface were studied using Monte Carlo simulations of a hexagonal lattice model, parameterised using DFT calculations. The parameters included molecule-surface interactions, that accounted for chemisorbed and physisorbed states, and molecule-molecule interactions.

In the $\mathrm{MC}$ simulations, it was observed that at low temperature $\mathrm{C}_{6} \mathrm{Cl}_{6}$ and $\mathrm{C}_{6} \mathrm{Br}_{6}$ reached ordered packed structures, due to their preference for the mobile physisorbed state. As the temperature is increased they undergo a series of phase transitions to a disordered packed physisorbed structure, a non-packed physisorbed layer and finally a non-packed mixed chemisorbed and physiorbed layer. Different behaviour is observed for benzene and $\mathrm{C}_{6} \mathrm{~F}_{6}$. At high temperature these molecules can access the physisorbed state and diffuse on the surface, 
whereas at low temperatures the molecules quickly become chemisorbed (their preferred adsorption state), which inhibits diffusion across the surface and the formation of ordered structures. Hence, benzene and $\mathrm{C}_{6} \mathrm{~F}_{6}$ remain in disordered arrested phases and do not form packed configurations along the whole temperature range explored.

The difference in energy between the chemi- and physisorbed states and interaction between physisorbed molecules are the main factors that control the self-assembly. However, changing the interactions between chemi- and physisorbed molecules also affects the stability of the different phases. For $\mathrm{C}_{6} \mathrm{Cl}_{6}$ and $\mathrm{C}_{6} \mathrm{Br}_{6}$ as this interaction becomes stronger the formation of ordered monolayers is seen at slightly higher temperatures. Varying the interactions between two chemisorbed molecules has no effect on the structures formed, since once molecules are chemisorbed they are unable to move to form ordered structures.

Due to the assumptions adopted in the model a number of processes observed in experimental systems, such as desorption or dissociation, have not been accounted for. For halogenated benzene molecules on $\mathrm{Pt}(111)$ these typically occur at temperatures above room temperature. Studies have shown that benzene exhibits two chemisorption temperatures, at $320 \mathrm{~K}$ and $425 \mathrm{~K}$ [40], and that it desorbs from Pt(111) between $300 \mathrm{~K}$ and $500 \mathrm{~K}$, with dehydrogation occuring above $400 \mathrm{~K}$ [41]. Only for bromobenzene, is dissociation on $\operatorname{Pt}(111)$ observed at low temperatures of $200 \mathrm{~K}$ [42]. It should be understood that the phase transition temperature in lattice gas models is always highly overestimated due to the reduction of possible states in the discrete lattice model, compared to the real experimental situation where there are many intermediate states. Nevertheless, it is expected that the structures observed in this work should hold at low temperatures. This means that it is impossible that benzene and $\mathrm{C}_{6} \mathrm{~F}_{6}$ to form packed patterns, whereas $\mathrm{C}_{6} \mathrm{Cl}_{6}$ and $\mathrm{C}_{6} \mathrm{Br}_{6}$ will remain physisorbed and form packed patterns with different molecule ordering at low temperatures.

This model captures a key aspect of the interplay between molecule-molecule and molecule-surface interactions by including both chemisorbed and physisorbed states. This feature has been previously overlooked when modelling the collective behavior of surfaceadsorbed molecular monolayers. Future directions of this work are to account for multiplicity of adsorption sites and include directionality in the molecule-molecule interactions to account for molecules with different symmetries and number of halogen atoms. We propose that functionalising molecules in order to tune their adsorption states, is an innovative way to control monolayer structure and will lead to the ability to form new 2D structures, building 
on current work on graphene nanoribbons.

\section{ACKNOWLEDGEMENT}

We acknowledge the Academia Nazionale dei Lincei and the Royal Society of Edinburgh for financial support.

[1] Seiki, N.; Shoji, Y.; Kajitani, T.; Ishiwari, F.; Kosaka, A.; Hikima, T.; Takata, M.; Someya, T.; Fukushima, T. Science 2015, 348, 1122.

[2] Bartels, L. Nature Chemistry 2010, 2, 87.

[3] Bieri, M.; Nguyen, M.-T.; Gröning, O.; Cai, J.; Treier, M.; Aït-Mansour, K.; Ruffieux, P.; Pignedoli, C. A.; Passerone, D.; Kastler, M.; Müllen, K.; Fasel, R. J. Am. Chem. Soc. 2010, 132, 16669-16676.

[4] Lafferentz, L.; Eberhardt, V.; Dri, C.; Africh, C.; Comelli, G.; Esch, F.; Hecht, S.; Grill, L. Nature Chemistry 2012, 4, 215-220.

[5] Massimi, L.; Ourdjini, O.; Lafferentz, L.; Koch, M.; Grill, L.; Cavaliere, E.; Gavioli, L.; Cardoso, C.; Prezzi, D.; Molinari, E.; Ferretti, A.; Mariani, C.; Betti, M. G. J. Phys. Chem. C 2015, 119, 2427-2437.

[6] Heimel, G.; Duhm, S.; Salzmann, I.; Gerlach, A.; Strozecka, A.; Niederhausen, J.; Bürker, C.; Hosokai, T.; Fernandez-Torrente, I.; Schulze, G.; Winkler, S.; Wilke, A.; Schlesinger, R.; Frisch, J.; Bröker, B.; Vollmer, A.; Detlefs, B.; Pflaum, J.; Kera, S.; Franke, K. J.; Ueno, N.; Pascual, J. I.; Schreiber, F.; Koch, N. Nature Chemistry 2013, 5, 187-194.

[7] Skomski, D.; Jo, J.; Tampas, C. D.; Kim, S.; Lee, D.; Tait, S. L. Langmuir 2014, 30, 1005010056.

[8] Fortuna, S.; Cheung, D. L.; Troisi, A. J. Phys. Chem. B 2010, 114(5), 1849-1858.

[9] Misiūnas, T.; Tornau, E. E. J. Phys. Chem. B 2012, 116(8), 2472-2482.

[10] Ibenskas, A.; Tornau, E. E. Phys. Rev. E 2012, 86, 051118.

[11] Šimėnas, M.; Tornau, E. J. Chem. Phys. 2013, $139(15), 154711$.

[12] Szabelski, P.; Rżysko, W.; Pańczyk, T.; Ghijsens, E.; Tahara, K.; Tobe, Y.; De Feyter, S. RSC Advances 2013, 3(47), 25159-25165. 
[13] Lin, T.; Wu, Q.; Liu, J.; Shi, Z.; Liu, P. N.; Lin, N. J. Chem. Phys. 2015, 142(10), 101909.

[14] Rżysko, W.; Patrykiejew, A.; Binder, K. Phys. Rev. B 2005, 72, 165416.

[15] Fefelov, V. F.; Gorbunov, V. A.; Myshlyavtsev, A. V.; Myshlyavtseva, M. D. Phys. Rev. E 2010, 82, 041602.

[16] Roussel, T. J.; Vega, L. F. J. Chem. Theory Comput. 2013, 9(5), 2161-2169.

[17] Björk, J.; Hanke, F.; Stafström. J. Am. Chem. Soc. 2013, 135, 5768-5775.

[18] Wong, S. L.; Huang, H.; Huang, Y. L.; Wang, Y. Z.; Gao, X. Y.; Suzuki, T.; Chen, W.; Wee, A. T. S. J. Phys. Chem. C 2010, 114(20), 9356-9361.

[19] de Oteyza, D. G.; Wakayama, Y.; Liu, X.; Yang, W.; Cook, P. L.; Himpsel, F. J.; Ortega, J. E. Chem. Phys. Lett. 2010, 490(1-3), 54-57.

[20] de Oteyza, D. G.; El-Sayed, A.; Garcia-Lastra, J. M.; Goiri, E.; Krauss, T. N.; Turak, A.; Barrena, E.; Dosch, H.; Zegenhagen, J.; Rubio, A.; Wakayama, Y.; Ortega, J. E. J. Chem. Phys. 2010, 133(21).

[21] Makoudi, Y.; Baris, B.; Jeannoutot, J.; Palmino, F.; Grandidier, B.; Cherioux, F. Chem. Phys. Chem. 2013, 14, 900-904.

[22] Copie, G.; Makoudi, Y.; Krzeminski, C.; Cheŕioux, F.; Palmino, F.; Lamare, S.; Grandidier, B.; Cleri, F. J. Phys. Chem. C 2014, 118, 12817.

[23] Copie, G.; Cleri, F.; Makoudi, Y.; Krzeminski, C.; Berthe, M.; Cherioux, F.; Palmino, F.; Grandidier, B. Phys. Rev. Lett. 2015, 114, 066101.

[24] Wander, A.; Held, G.; Hwang, R.; Blackman, G.; Xu, M.; de Andres, P.; Hove, M. V.; Somorjai, G. Surf. Sci. 1991, $249(1-3), 21-34$.

[25] Yau, S.-L.; Kim, Y.-G.; Itaya, K. J. Am. Chem. Soc. 1996, 118(33), 7795-7803.

[26] Yau, S.-L.; Kim, Y.-G.; .; Itaya, K. The Journal of Physical Chemistry B 1997, 101(18), 3547-3553.

[27] Liu, W.; Filimonov, S. N.; Carrasco, J.; Tkatchenko, A. Nat. Commun. 2013, 4, 2569.

[28] Peköz, R.; Johnston, K.; Donadio, D. J. Phys. Chem. C 2014, 118(12), 6235-6241.

[29] Peköz, R.; Johnston, K.; Donadio, D. J. Phys. Chem. C 2012, 116, 20409.

[30] Landau, D.; Binder, K. A guide to Monte Carlo Simulations in Statistical Physics; Cambridge University Press: Cambridge, 2000.

[31] Johnston, K.; Peköz, R.; Donadio, D. Surf. Sci. 2016, 644, 113-121.

[32] Johnston, K.; Kleis, J.; Lundqvist, B. I.; Nieminen, R. M. Phys. Rev. B 2008, 77, 121404(R). 
[33] Kim, H.-J.; Tkatchenko, A.; Cho, J.-H.; Scheffler2, M. Phys. Rev. B 2012, 85, 041403.

[34] Giannozzi, P.; Baroni, S.; Bonini, N.; Calandra, M.; Car, R.; Cavazzoni, C.; Ceresoli, D.; Chiarotti, G. L.; Cococcioni, M.; Dabo, I.; Corso, A. D.; de Gironcoli, S.; Fabris, S.; Fratesi, G.; Gebauer, R.; Gerstmann, U.; Gougoussis, C.; Kokalj, A.; Lazzeri, M.; Martin-Samos, L.; Marzari, N.; Mauri, F.; Mazzarello, R.; Paolini, S.; Pasquarello, A.; Paulatto, L.; Sbraccia, C.; Scandolo, S.; Sclauzero, G.; Seitsonen, A. P.; Smogunov, A.; Umari, P.; Wentzcovitch, R. M. J. Phys.: Condens. Matter 2009, 21, 395502.

[35] Dion, M.; Rydberg, H.; Schröder, E.; Langreth, D. C.; Lundqvist, B. I. Phys. Rev. Lett. 2004, 92, 246401.

[36] Thonhauser, T.; Cooper, V. R.; Li, S.; Puzder, A.; Hyldgaard, P.; Langreth, D. C. Phys. Rev. $B$ 2007, 76, 125112.

[37] Roman-Periz, G.; Soler, J. M. Phys. Rev. B 2009, 103, 096102.

[38] Puzder, A.; Dion, M.; Langreth, D. C. J. Chem. Phys. 2006, 124, 164105.

[39] Krauss, T. N.; Barrena, E.; Dosch, H.; Wakayama, Y. Chem. Phys. Chem. 2009, 10, 24452448 .

[40] Marsh, A. L.; Gland, J. L. Surf. Sci. 2003, 536, 145-154.

[41] Lee, A. F.; Chang, Z.; Hackett, S. F. J.; Newman, A. D.; Wilson, K. J. Phys. Chem. C 2007, 111(28), 10455-10460.

[42] Haines, B. M.; Gland, J. L. Surf. Sci. 2008, 602, 1871-1876. 